\title{
Virtualized Interoperability Testing: Application to IPv6 Network Mobility
}

\author{
Ariel Sabiguero, Anthony Baire, Antoine Boutet, and César Viho \\ Institut de Recherche en Informatique et Systèmes Aléatoires \\ Campus Universitaire de Beaulieu \\ 35042 Rennes CEDEX, France \\ asabigue@fing.edu.uy, \{asabigue, abaire, aboutet, viho\}@irisa.fr,
}

\begin{abstract}
Interoperability testing is an inherently distributed task. It connects different implementations together and determines if they interact according to their specifications, providing the expected services.

Deployment, configuration management and operation of an interoperability solution can be drastically improved with the use of virtualization techniques. Virtualized solution allows simpler and more reliable testing, as less equipment is required and full automation is achieved, providing means for better testing.
\end{abstract}

Keywords: Interoperability Testing, virtualization, management, reliability, cost.

\section{Introduction}

Interoperability testing is a discipline that requires deployment of several pieces of equipment. The way it addresses the verification of required properties in implementations is based on populating configurations with existing implementations and making them interwork.

In this work we present a solution based on the combined use of network virtualization and machine virtualization. The solution solves management problems that allows us to deploy several configuration scenarios with fixed hardware configurations. The operation of the virtualized test platform not only solves technical issues that previously were only addressed with inaccurate physical manipulations, but saves resources and time. Complete testbeds involving up to seven devices and five networks can be virtualized into a single physical host. The field of application is IPv6 Network Mobility testing following test specifications from IPv6 Ready Logo, an international certification program.

The IPv6 Network Mobility (NEMO) Basic Support protocol specification can be found in the Request For Comments (RFC) 3963 [1. It is an extension to the Mobile IPv6 protocol and enables the support for the network mobility. This extension allows session continuity and reachability for every node in the Mobile Network as the network moves. The protocol is designed so that network mobility is transparent to the nodes inside the Mobile Network. 


\section{Virtualized solution description}

This section introduces the building blocks used in the construction of the virtualized environment. Technical details of configuration are beyond the scope of this work. Despite of that, we believe that a test engineer can deploy an equivalent platform with provided information.

\section{$2.1 \quad$ Network Virtualization}

Network Virtualization is the first step for deploying a virtualized test environment. This is a critical step since the network is the central component for making implementations interact with each other. Virtualized network shall not introduce any bias in the interactions while still allowing observability.

Our approach uses Virtual LANs defined by the IEEE 802.1Q standard 2] and software bridges. An intermediate solution, proposed in [3], introduced a partially virtualized network made of a Linux box and an Ethernet switch. In the present work the complete network is deployed in the GNU/Linux box.

Each node taking part in the test is configured to use one or more network interfaces. Each interface is connected to the virtual network and mapped to a separate VLAN in the Linux host OS.

To interconnect interfaces, their corresponding VLANs are associated to software bridges. The software bridge is responsible for implementing the collision domain semantic and connecting these interfaces spanned through different VLANs.

We achieve required connection between components, and each element can communicate with others in the same way as they would using physical hardware components. Moreover all the traffic between the elements transit through the software bridges, this allows full observability. Traffic recording requirements are achieved by recording all the traffic directly on the Linux bridge devices.

\subsection{Machine Virtualization}

The main goal behind machine virtualization is to collapse a set of virtual nodes into a single physical one. Benefits range from optimized resource allocation to separation of concerns of servers and services. We use virtualization to cut the explosion of nodes required for interoperability testing.

The goal is to collapse $m$ virtual hosts into $n$ physical ones without introducing any bias in the verdict. We expect $m>>n$, and preferably, $n=1$. State of the art Network Mobility requirements are addressed from a single commodity PC.

For each node deployed on a virtual machine a set of network interfaces is configured. These devices are emulated by the VMware server. The guest system detect and accesses them like real physical interfaces and on the host system they are connected to their dedicated VLAN. The number of network interfaces depends on the role of the node in the test suite. A host node will require one interface for the test and a second interface for accessing the management network. If the node act as a router, then a third interface is needed. 


\subsection{Automated Virtualized Test Management}

Test management and networking are centralized on the host system. Executing a single test case consists of executing various actions sequentially. Actions can be executed directly on the host system and remotely on guest systems through the management network. Mobility events can be performed by the host system.

Test case operation sequences follow the usual three-stage arrangement: preamble, test body and postamble. The preamble contains actions for setting up the initial topology by configuring the network and nodes. The postamble contains the opposite actions for resetting the testbed to its original state. The test body contains the actions that addresses directly the property or properties being verified by the test purpose.

Mobility testing implies topology changes during the execution of the test body. These mobility events are a very specific requirements that disallows full automation when using non-virtualized network. With the virtualized solution the mobility events can be implemented as two successive actions: mobile router VLAN is removed from the first bridge and added to the second bridge.

Any topology change can be automaticaly integrated in the test sequence and interleaved with other actions without requiring manual intervention. This reduces drastically the risk of network configuration errors (connecting a cable to the wrong network) as well as synchronization errors (not connecting a cable at the right time in the test sequence). Reliability and accuracy of test execution is enhanced, as field observations described in the next section.

\section{$3 \quad$ Field Results}

This section present results from field experience together with laboratory results. Not all the technologies used in our proposal are already accepted for certification purposes, thus, we can only present laboratory results.

\subsection{Field Error Analysis}

Manual operations are still required, and might always be required when there is no possibility to automate the IUT (Implementation Under Test). The conventional platform used for testing consists of several hubs, in which the different nodes are plugged test after test. Using IPv6 Ready Logo accepted technologies and practices, we studied the source of errors encountered during test execution.

Presented methodology completely remove network configuration errors, which account for almost one quarter of the field errors. A solution that automates the IUT fully solves synchronization, disappearing the source of errors too, removing up to $50 \%$ of total errors in that case.

\subsection{Execution Time, Cost and Reliability}

Figures presented here were gathered during real interoperability events and in laboratory. The addition of the management network, which solved the synchronization overhead and errors amongst the nodes of the test platform. The 
first solution was manually synchronized, even though automated and average times for test suite execution exceeded four hours -no time execution data was gathered with that tool-. With the addition of the parallel network, average execution times shrank to 2:50hs. Despite of that, some test executions might take up to 4:55hs. This is due to complexity of operation of the IUT or presence of unforseen problems. Due to this fact, when interoperability events are scheduled only two devices per day are scheduled, per test platform and test engineer. In laboratory executions, where conditions are better controlled, execution time averages 2:05hs.

The solution and methodology required by the IPv6 Ready Logo involves up to 7 test nodes, the test manager node and 5 network hubs are required. Presented solution can be completely collapsed inside a single physical computer, avoiding all network complexity and using standard Ethernet interfaces to connect to the IUT. State of the art hardware can handle the whole workload, thus the cost saving ratios can be estimated between 5:1 to 10:1.

Reliability of the collapsed testbed is drastically enhanced. Initial solution requires various equipments, the probability of failure is not negligible and increases accordingly the number of components. Reliability becomes more relevant in international interoperability events, where the complete platform has to be commuted. Transporting seven notebooks might sometimes lead to a broken node, leading to a non operational platform.

\section{Conclusions}

Presented methodology solves several problems found executing abstract test specifications for interoperability testing. The solution was successfully implemented. Experimental results show that execution errors due to unreliability of wireless technologies were removed without loosing automation. Complete virtualized environment for interoperability mobility testing in a single box provides cost savings and deployment facilities that simplifies execution and testing operations. Virtualization proved to be applicable to IPv6 in general and mobility testing in particular. Virtualization optimizes existing practices considering cost, reliability and fault tolerance of the testbed without introducing bias in the verdicts.

\section{References}

1. Devarapalli, V., Wakikawa, R., Petrescu, A., Thubert, P.: RFC 3963: Network Mobility (NEMO) Basic Support Protocol (January 2005)

2. IEEE Standards for Local and Metropolitan Area Networks: Virtual Bridged Local Area Networks, IEEE Std.802.1q (1998)

3. Sabiguero, A., Viho, C.: Plug once, test everything. Configuration Management in IPv6 Interop Testing. In: Proceedings of the Fifteenth Asian Test Symposium. IEEE Computer Society Conference Publishing Services, pp. 443-448. IEEE Computer Society, Los Alamitos (2006) 